\title{
Computational Study on the Electrical Behavior of Silicon Nanowire Memristive Biosensors
}

\author{
Ioulia Tzouvadaki, Francesca Puppo, Marie-Agnès Doucey, Giovanni De Micheli, Fellow, IEEE, \\ and Sandro Carrara
}

\begin{abstract}
In this paper, a complete study is carried out investigating the relationship between the biosensing and the electrical characteristics of freestanding two-terminal Schottky-barrier silicon nanowires. This paper successfully reproduces computationally the electrical behavior obtained experimentally from the nanowire devices before and after the surface biomodification. Throughout modeling and simulations, this paper confirms that the experimental results obtained from the electrical characterization of bare two-terminal Schottky-barrier silicon nanowires present current-to-voltage characteristics fully equivalent to that of a pure memristor device, according to the literature. Furthermore, this paper shows that the voltage gap appearing in the current-to-voltage characteristics for nanowires with biomodified surface is related to capacitive effects due to minority carriers in the nanowire and it is also indicated that those effects are strongly affected by the concentration of antigens uptaken on the device surface. Overall, this paper confirms the implication of the memristive effect for biosensing applications and therefore, demonstrates the memristive biosensors.
\end{abstract}

Index Terms-Biosensor, memristor, silicon nanowire, Schottky barrier, antigen uptake.

\section{INTRODUCTION}

$\mathbf{P}$ RONOUNCED hysteresis in electrical characteristics is a unique and important signature of memory devices [1]. The hysteresis appearing in the current to voltage characteristics has been attributed to a wide range of phenomena, including molecular redox events, metal filament formation and destruction. Indicatively as mentioned by Agapito et al. [2] who studied the atomistic origin of the memristive-switching mechanism using quantum-mechanical atomistic calculations, the current to voltage characteristics reported are associated with solid-state redox reactions with dynamic doping modifying the conductance of the channel or with abrupt ON/OFF transitions where the mechanism depends

Manuscript received June 6, 2015; revised June 26, 2015; accepted July 7, 2015. Date of publication July 14, 2015; date of current version August 26, 2015. This work was supported in part by the PROSENSE Project through the European Union's Horizon 2020 Research and Innovation Programme under Grant 317420 and in part by the Multidisciplinary FNS under Grant CR32I3 135073. The associate editor coordinating the review of this paper and approving it for publication was Prof. Istvan Barsony.

I. Tzouvadaki, F. Puppo, G. De Micheli, and S. Carrara are with the Integrated System Laboratory, École Polytechnique Fédérale de Lausanne, Lausanne 1015, Switzerland (e-mail: ioulia.tzouvadaki@epfl.ch; francesca. puppo@epfl.ch; giovanni.demicheli@epfl.ch; sandro.carrara@epfl.ch).

M.-A. Doucey is with the Ludwig Center for Cancer Research, University of Lausanne, Lausanne 1015, Switzerland (e-mail: marie-agnes.doucey@unil.ch).

Color versions of one or more of the figures in this paper are available online at http://ieeexplore.iee.org.

Digital Object Identifier 10.1109/JSEN.2015.2456336 on bias voltage-induced formation and breaking of conductive atomic filaments.

Furthermore, memory effects are reported due to the finite mobility of ions as they redistribute within a charged nanopore under applied potentials and more specifically due to non-instantaneous ion redistribution [3]. Thus, these effects are considered to originate from the dynamic properties of charge carriers (electron or ions) [4], [5].

In 2008 Strukov et al. [6] introduced the first physical implementation of a memristor consisting of a two-layer thin film of $\mathrm{TiO}_{2}$, the one of them doped with oxygen vacancies, sandwiched between platinum contacts. The model can be considered as a simple analytical example that memristance arises naturally in nanoscale systems in which solid-state electronic and ionic transport are coupled under an external bias voltage. The dopants don't stay stationary in a high electric field and tend to drift in the direction of the current. Putting a bias voltage across a thin film of $\mathrm{TiO}_{2}$ semiconductor that has dopants only on one side causes them to move into the pure $\mathrm{TiO}_{2}$ on the other side and thus lowers the resistance. Running current in the other direction will then push the dopants back into place, increasing the resistance.

Memristive devices have already been used in many applications. Plethora of applications have been suggested in the fields of logic design and memory issues, since memristors enable new possibilities for computation and non-volatile memory storage. Indicatively, the construction of memristorbased digital logic circuits has been introduced [7] where memristors are explored for their potential use in dense programmable logic circuits. In addition, applications concerning artificial synapses have been suggested [8]-[11] where a memristor emulator realizes all required synaptic properties and is introduced to emulate the biophysics and temporal dynamics of real synapses. Furthermore, it is worth mentioning the introduction of the Resistive RAM memories [12] which can be classified as memristors and can be considered suitable candidates for standalone memory applications due to the excellent scalability and programming time that can be obtained comparing to conventional Flash memories. Generic Memristive Structure for 3-D FPGA applications has been proposed [13] too, which can be utilized for steering logic that is useful for multiplexing signals and therefore capable in replacing the traditional pass-gates in FPGAs.

According to literature [14], semiconductor nanowires and semiconducting carbon nanotubes have been reported as 
promising building blocks for future biosensors, enabling direct electrical detection of biomolecules. Ishikawa et al. [15] also introduced a nanowire device functionalized with engineered proteins possessing elevated affinity toward target molecules, demonstrating the nanowire based biosensors applicability as an accurate and important method to measure the dissociation constants for biological complex systems.

Although the fact that memristive effect had already been introduced in so many different applications, it is only recently that it has been suggested and applied for biosensing purposes taking advantage of the electrical characteristics of memristive silicon nanowire devices [16], [17]. Furthermore, in another recent application, memristive silicon nanowire devices are used as $\mathrm{pH}$ sensors demonstrating the possibility of sensing $\mathrm{pH}$ variations via the detection of a different amount of hydrogen ions of a salt solution deposited and dried over the memristive $\mathrm{pH}$ sensor [18].

Throughout the works introduced by Sacchetto et al. [16] and Carrara et al. [17] Schottky-barrier silicon nanowire devices functionalized with antibody films and exhibiting memristive behavior are used as biosensors and target at the detection of biomarkers. More specifically, the hysteresis loop of the current to voltage characteristics of the devices provides a new approach in biodetection, sensing the presence of biomarkers achieving a low-cost label-free detection of biological processes in dry conditions. For bare nanofabricated wires, the current to voltage characteristics indicate pinched hysteretic loop at zero voltage. In these devices, the memory effect depends on charge carrier rearrangement at the nanoscale as due to external perturbation. However, the pinched hysteresis appears shifted from zero voltage to different voltage values when biological substances are present on the device surface. Namely, a voltage gap is created in the semi-logarithmic current to voltage curve after the nanowire bio-modification as a further memory effect on the voltage scan across the nanowire [17].

The presence of biological substances all-around the freestanding nanowire contributes to extra charges surrounding the device and the net contribution of their charged residues act by creating an electrical field surrounding the channel of the nanowire device, resulting to an effect equivalent to that obtained in case of nanostructures without any bio-functionalization but fabricated with an all-around silicon gate [17]. The all-around gate gives to those structures this particular memristive conductivity, which includes the presence of a voltage shift in the electrical characteristics.

The present work aims to present a model based on equivalent electrical circuits to study the electrical behavior of bare freestanding two-terminal Schottky-barrier silicon nanowire devices, as well as, that of bio-functionalized freestanding two-terminal Schottky-barrier silicon nanowire devices, namely memristive biosensors. The paper also aims to provide an understanding of the effects occurring to the electrical characteristics after the bio-functionalization process and the uptake of antigens on the device surface. The results of this work validate computationally the memristive behavior exhibited by the nanowire devices and demonstrate that the behavior of the physical system with bio-modified surface is related to capacitive effects owing to the minority carriers in the nanowire device and that those effects are affected by the concentration of antigens uptaken on the device surface, evidence that demonstrates the efficiency of the memristive biosensor.

\section{Materials AND Methods}

\section{A. Experimental Methodology}

1) Fabrication of Silicon Nanowire Devices: Schottkybarrier Silicon Nanowire devices are fabricated through a top-down fabrication process performed on Silicon-On-Insulator wafers. Commercially available (100) oriented Silicon-On-Insulator wafers with low boron concentration $\left(\mathrm{N}_{\mathrm{A}} \approx 10^{15}\right.$ atoms $\left./ \mathrm{cm}^{3}\right)$ are used in this procedure. The wafer is first coated with poly(methyl methacrylate), a standard positive resist for Electron-Beam Lithography, and then exposed to an electron beam source to pattern the first mask for metal contacts. After resist development, the wafer is cleaned in a buffer HF etching bath in order to remove any trace of native oxide formed on the exposed areas of the wafer. A 30nm-thick layer of Nickel is then evaporated onto the device, and dissolved in acetone. The lift-off process leads to the definition of Nickel contact regions. Nickel silicidation is then obtained by annealing performed by successive exposition of 20 minutes in forming gas at $200^{\circ} \mathrm{C}, 300^{\circ} \mathrm{C}$ and $400^{\circ} \mathrm{C}$, respectively. Nickel silicidation is performed in order to form the Schottky-barrier junctions of the device and the NiSi contact pads for electrical characterization. A layer of Hydrogen SilselsQuioxane, negative tone resist for Electron-Beam Lithography, is then spin-coated on top of the wafer and patterned into lines by an Electron-Beam Lithography process. Deep Reactive Ion Etching is used to produce a scalloped trench in a monocrystalline silicon substrate. Repeated Deep Reactive Ion Etching cycles lead to verticallystacked, free-standing silicon nanowires anchored between two NiSi pillars.

Morphological analysis of the memristive sensor is carried out using a Scanning Electron Microscopic MERLIN, from Zeiss. The fabricated silicon nanowire devices are of a length of $420 \mathrm{~nm}$ and a width of $38.4 \mathrm{~nm}$. Elfström et al. [14] analyzed the size dependence of the sensitivity of Silicon nanowires of different widths fabricated in silicon on insulator material, for biomolecule detection and for other sensor applications.

The aforementioned study revealed that using nanowires of a small diameter i.e. below $50 \mathrm{~nm}$, results to electrical properties dependent on the local environment where charge changes on the surface induce a field effect that significantly changes the carrier concentration and consequently the conductance. These findings confirm that the presence of charges on the surface as well as interface properties finally play a pivotal role to the channel's conductance of the fabricated silicon nanowire devices under study.

Morphological Scanning Electron Microscopic images acquired from one of the fabricated silicon nanowires are reported (Figure 1). A top view of the silicon nanowire is shown (Figure 1a), demonstrating the very small sizes 

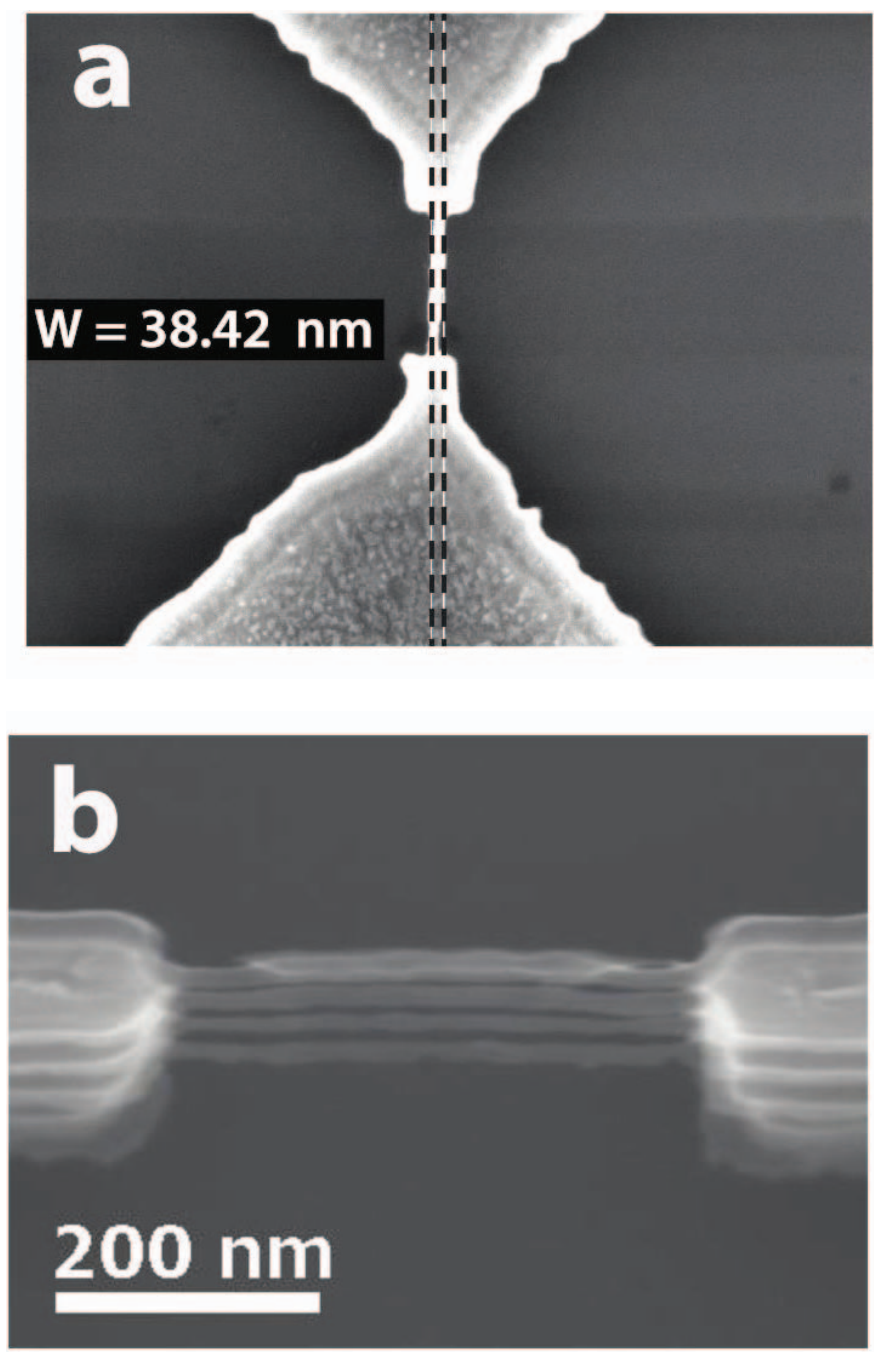

Fig. 1. (a) Top view of the silicon nanowire device. (b) Tilted view of the silicon nanowire device.

achieved with the top-down fabrication process described previously. In addition a tilted view of the same device is illustrated (Figure 1b), and shows the vertically-stacked structure of free-standing nanowires.

2) Surface Bio-Functionalization and Antigen Uptake: The fabricated memristive silicon nanowires are first cleaned in a piranha solution $\left(1: 1, \mathrm{H}_{2} \mathrm{O}_{2}: \mathrm{H}_{2} \mathrm{SO}_{4}\right)$ in order to clean the sample surfaces from any organic residues as well as to generate more surface hydroxyl terminating groups. In order to obtain the memristive biosensors, the silicon nanowires are functionalized by exposing the surface to GPTS solution (1\% in ethanol containing $10 \mathrm{mM}$ acetic acid) for $60 \mathrm{~min}$ at Room Temperature, followed by extensive rinsing in ethanol with $10 \mathrm{mM}$ acetic acid to remove unreacted GPTS. A self-assembled monolayer with terminal amino groups is then obtained by heating the sample at $110^{\circ} \mathrm{C}$ in a dry oven for $15 \mathrm{~min}$. Antibody attachment on the silicon nanowire surface is carried out by exposing the nanowire surface to $0.5 \mathrm{mg} / \mathrm{ml}$ antibody (anti-rabbit and anti-VEGF are used separately in different experiments) in $0.01 \times \mathrm{PBS}$ at Room Temperature overnight. The devices are then thoroughly washed with the same buffer. In order to prevent nonspecific binding of proteins in the detection measurement, the remaining active GPTS-derived groups are passivated by applying $10 \mathrm{mM}$ ethanolamine solution in PBS at Room Temperature for $1 \mathrm{~h}$, followed by washing with the same buffer. A further blocking step is carried out by an additional incubation of $30 \mathrm{~min}$ in PBS containing 3\% gelatin from cold-water fish skin. The modified surface is then washed with same solution and stored in PBS at $4^{\circ} \mathrm{C}$ until use.

The bio-functionalized devices are exposed to antigens (against anti-rabbit antibodies) for $2 \mathrm{~h}$ at room temperature in PBS. The excess of antigen is removed by washing the substrates surface three times with PBS and then the surface is dried using nitrogen flow. Every time, each device is measured first non bio-functionalized, after the bio-functionalization and then after the further bio-modification of the surface with antigen uptake at different concentrations.

3) Sensing Methods: Antibody-antigen coupling events are detected by measuring the current to voltage characteristics of the sensors in air on dried samples with the use of a Cascade Microtech Probe Station and a Hewlett-Packard 4165A Precision Semiconductor Parameter Analyzer. The measurements are carried out at Room Temperature, and the humidity is kept constant while testing, using the feedback from the Rotronic $\mathrm{HC}_{2}-\mathrm{CO}_{4}$ Thermo-Hygrometer sensor. For sensing measurement, a drain to source voltage sweep is performed forward and backward in the range $-3 \mathrm{~V}$ to $+3 \mathrm{~V}$. The back-gate potential $V_{B G}$ is kept grounded during the acquisition. A reference measurement is first applied on the fabricated structures just after the nano-fabrication process, followed by electrical monitoring of silicon nanowire devices functionalized with antibodies to get the baseline current and then, real sensing measurements on silicon nanowire devices after uptake of differently concentrated antigen solutions. The silicon nanowire devices are stored in fridge after the functionalization with antibodies. Afterwards, the devices are removed from the storage PBS solution and gently dried under $\mathrm{N}_{2}$ flow before been inserting in the measurement chamber. For testing the effect of antigen on the voltage gap, electrical data is acquired after following $1 \mathrm{~h}$ incubations at Room Temperature of the device in solutions of different antigen concentrations in $0.001 \times \mathrm{PBS}$. After each incubation the sample is thoroughly rinsed with the same buffer to eliminate unreacted antigen molecules and dried under a gentle stream of nitrogen. What follows is the insertion of the sample in the characterization tool and measurements in air in a controlled humid environment. Testing in dry conditions is preferred because a higher sensitivity is demonstrated due to the increased Debye screening length when the bulk solution is removed [17]-[19].

\section{B. Computational Methodology}

A memristor macromodel is designed and created using CADENCE (R) OrCAD Capture and Capture CIS software. Equivalent circuits are introduced consisting of the memristor macromodel in combination with analog circuit elements targeting at emulating the behavior of the physical system and studying the resulting electrical characteristics. The model aims to be a link between the theory concerning the memristors 
and the experimental aspect, opening new perspectives for biosensing applications.

1) Memristor Modeling: Due to the wide application possibilities that memristor devices may offer there have been efforts to study the memristive behavior experimentally as well as computationally [5], [6], [20]-[25]. Besides theoretical aspects and experiments, models, which approximate well the physical realization, are needed. In this framework it is worth mentioning the development of a simple compact model for representing the electrical behavior of memristors introduced by Biolek et al. [23] describing a mathematical SPICE model of the prototype of memristor, manufactured in 2008 in Hewlett-Packard (HP) Labs [6]. Furthermore in their work Rak and Cserey [24] create a memristor element in SPICE which simulates the published memristor realization introduced by HP Labs and offers the possibility to be used as a circuit element in design work.

In the present work a macromodel of a memristor element is created as inspired by Rak and Cserey [24] and Biolek et al. [23], [25]. The macromodeling of the memristor is based on the theoretical memristor introduced by Chua [26], Chua and Kang [27], and Chua [28] and Strukov et al. [6] and Williams [29] and is realized by using CADENCE (R) OrCAD Capture and Capture CIS software. OrCAD Capture enables schematic design entry for analog simulation and modeling of circuit operation using SPICE.

It is worth mentioning that the purpose of the present work is not to present a new design for modeling the electrical behavior of a pure memristor but to use the pure memristor as a building block to emulate more complex devices for biosensing purposes.

2) Memristor Equivalent Circuit: Design and Simulations: The memristor macromodel is considered as reference point and it is combined to analog circuit elements. Therefore, equivalent circuits are designed consisting of the memristor macromodel and analog circuit elements connected in series and in parallel in order to study the resulting electrical characteristics and emulate the different behaviors of the physical system. All simulations are carried out in the CADENCE (R) electronic design software. For simulating the behavior of a memristor an equivalent circuit is proposed, consisting of a sinusoidal input Voltage (Vin) source, the memristor element $(\mathrm{M})$ and a resistor $(\mathrm{R})$ required to allow the current flow. All the circuit elements are connected in series.

\section{RESULTS AND DISCUSSION}

\section{A. Electrical Characteristics}

Simulations have been implemented for the proposed equivalent circuit simulations under a sinusoidal input voltage. The input voltage frequency value is chosen accordingly to the values widely presented in earlier and current works concerning the memristors study in the range of $[100-1000 \mathrm{~Hz}]$ and the input voltage amplitude is selected to match the experimental set up input values. The current to voltage results obtained by the simulations depict the characteristic pinched hysteresis loop at zero voltage validating qualitatively and quantitatively the functionality of the model and confirming its ability in properly and adequately simulating a memristor. The obtained electrical characteristics seem to be a good approximation of the measurement of the real memristor [6], [26]-[28] confirming the fact that the model actually describes the operation of a memristor, and validating the creation of a functional memristor model according to theory [26]-[28].

\section{B. Frequency Dependence}

The effect of the different frequencies in the current to voltage characteristics is also studied. Varying the input voltage frequency does not affect the memristive behavior but it modifies the shape of the memristive curve accordingly to previous literature [6], [26]-[28]. The memristive effect diminishes as the frequency is increasing and the characteristics become closer to linear. This effect is the direct consequence of dynamics and it is physically reasonable too by taking into account the finite charge mobility in the device [23]-[25]. Simulations are carried out for different frequencies values and confirm this behavior (data not shown).

\section{Equivalent Circuits for Matching Experimental Results}

The modeling and simulations through the proposed equivalent circuits may be considered as a link between the theoreti$\mathrm{cal}$ and the experimental aspect where the model must have the ability to reproduce and emulate computationally the behavior of the physical system regarding the electrical characteristics.

In order to understand the experimental results and to draw conclusions regarding the absence of biological substances on the nanowire surface, semi-logarithmic range for the current with respect to input voltage curve is of the most significant interest and better highlights the features that characterize the device.

The corresponding semi-logarithmic current to voltage curve for the pure memristor model (Figure 2), obtained through simulation of the initial equivalent circuit, modeling the pure memristor device. As expected, it can be noticed that the two local minima for the forward and the backward regimes of the voltage sweeps occur for the same input voltage value and more specifically for zero input voltage $(\operatorname{Vin}=0)$.

It can be observed that the experimentally acquired curve (Figure 2) and the curve obtained from simulating the memristor model (Figure 2), present a significant resemblance. To this end, achieving the best fitting with the experimental results requires model refinements thought additional analog circuit elements that must be introduced to the initial equivalent circuits for modifying the obtained memristive curves. In addition the values of the circuit elements must be appropriately estimated in order to achieve the best fitting to the experimental results and elicit a proper physical interpretation.

1) Bare Two-Terminal Schottky-Barrier Silicon Nanowires (Memristive Devices): Metal contacts to bulk semiconductors usually result in Schottky barrier junctions with rectifying behavior [30]. According to previous works mentioned in literature [31]-[34] silicon nanowire FETs with Schottky source and drain contacts can be modeled as metal-semiconductormetal (M-S-M) structures with finite Schottky barrier heights. 

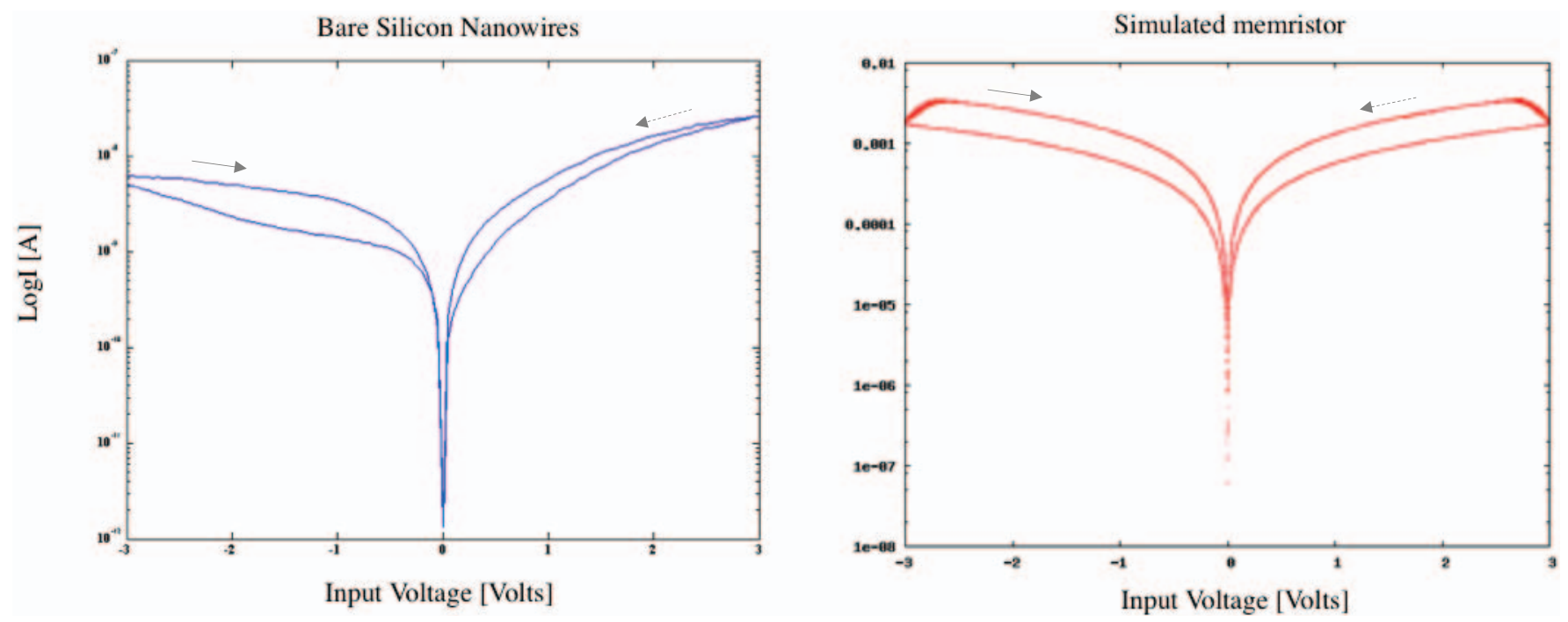

Fig. 2. Experimental (left) and Computational (right) semi-logarithmic current to voltage characteristic curve obtained from the electrical characterization of bare silicon nanowires and from the equivalent circuit for simulating the pure-memristive behavior respectively. The simulation voltage amplitude applied is 3 Volts and the frequency $700 \mathrm{~Hz}$. Arrows indicate forward (solid line) and backward (dashed line) regimes. The pinched hysteresis loop at zero voltage can be observed in both cases.

The modeling is based on equivalent circuits including a Schottky diode representing the metal-semiconductor (M-S) contacts and considering the nanowire as a resistor. Lee et al. [33] developed an equivalent circuit model, which consisted of one reverse-biased Schottky diode, one resistor, and one forward-biased Schottky diode connected in series. In this model, the intrinsic nanowire channel was modeled as one linear resistor and the gate voltage dependence of the nanowire was not included.

In addition there has also been introduced [31] a silicon nanowire FET model based on an equivalent circuit consisting of two Schottky diodes for the metal-semiconductor contacts and one MOSFET for the intrinsic silicon nanowire FETs. Furthermore, Elhadidy et al. [32] modeled the symmetrical, non-linear current to voltage characteristics of a metal-semiconductor-metal structure of two metallic Schottky contacts fabricated to a p-type semiconductor by treating the semiconductor as a resistor sandwiched between two identical head-to-head Schottky barriers. Each one of the two Schottky barriers is modeled as a sub-circuit consisting of a diode in parallel to a resistor.

In the present work an equivalent circuit model is developed by following the concept introduced by Elhadidy et al. [32] consisting of a memristor sandwiched between two identical head-to-head Schottky barriers. The Schottky barriers are represented by sub-circuits consisting of a diode in parallel to a resistor and result to a slight modification of the memristive curve. More specifically, the introduction of a diode to the initial system brings to the memristive curve the typical Schottky contact shape at the branches of the characteristic semi-logarithmic current to voltage curve (Figure 3). It is important to clarify that the location of the current minima is not affected since both occur for zero voltage for the forward and the backward regimes. A unique current corresponds to each applied voltage. If the polarity of the bias voltage is exchanged, the reverse-biased barrier would be exchanged

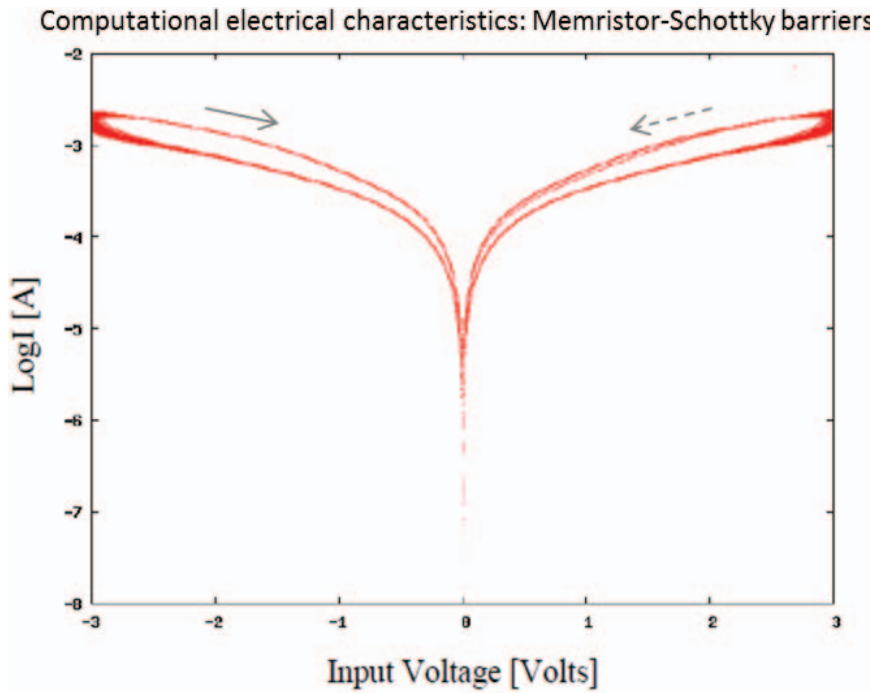

Fig. 3. Computationally obtained semi-logarithmic current to voltage results for the equivalent circuit including a memristor sandwiched between two identical head-to-head Schottky barriers. The memristive pinched hysteresis loop at zero voltage can still be clearly observed for both forward (solid line arrow) and backward (dashed line arrow) regimes.

with the forward-biased one and vice versa. For consistency reasons and interest, the input values of the sinusoidal Voltage (Vin) source are the same with respect to the case of the pure memristor equivalent circuit.

Experimental current to voltage characteristics present noticeable asymmetry at the branches of the semi-logarithmic current to voltage characteristics. Under ideal circumstances, the electrical characteristics in both branches of the semi-logarithmic current to voltage curve would be symmetrical since the Schottky barriers of the device structure are considered to be identical. Nevertheless, the measured data in real experimental conditions indicates non-identical branches in the semi-logarithmic electrical characteristics for 


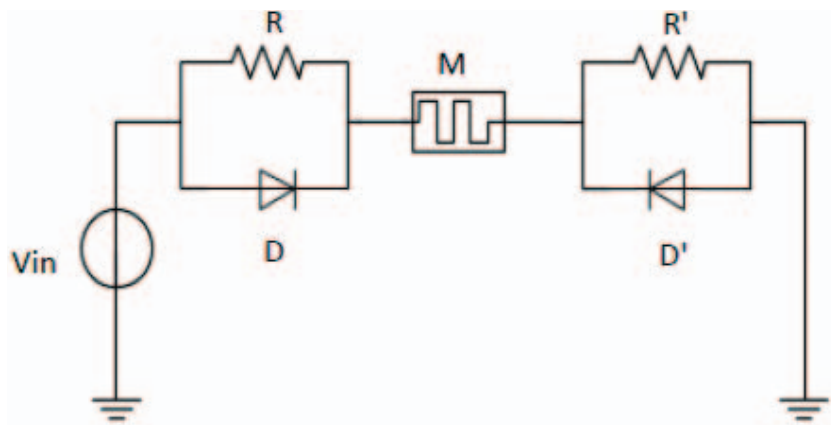

Fig. 4. Equivalent circuit of a memristor sandwiched between two non-identical head-to-head Schottky barriers. The sub-circuits consisting of a diode in parallel to a resistor emulate the effect of the Schottky barriers. The circuit consists of a sinusoidal input voltage source, resistances in the range of $0,5 \mathrm{k} \Omega-1 \mathrm{k} \Omega$ and common silicon epitaxial planar fast switching diodes provided by SPICE.

the majority of the devices under study. This slight difference in the branches asymmetry may be explained as a consequence of the non-identical area of contacts occurring in the real conditions, mainly due to the presence of the different interfacial insulating layers at both electrode contacts.

The concept of the non-identical Schottky barriers is taken into consideration in the equivalent electrical circuit (Figure 4) through $\mathrm{R}^{\prime}$ in combination to the fact that during the one circle of the current (depending on the polarity of the bias voltage) the one diode does not conduct due to the forward and reverse bias nature of the diode and consequently only the remaining resistivity origin from the reversed bias Schottky diode finally contributes. For consistency reasons and interest, the input values of the sinusoidal voltage source are the same with respect to the case of pure memristor equivalent circuit.

Simulation results obtained by the aforementioned equivalent circuit are compared to experimental data (Figure 5) namely, the experimental results obtained by the electrical characterization of bare silicon nanowire devices. The nanofabricated devices usually present a non-identical behavior by nature. It is demonstrated that the simulation curve follows in good approximation the average behavior of the physical system. According to this, it can be concluded that the experimental results present a current to voltage characteristic curve equivalent to that of a memristor device electrically contacted by two asymmetric Schottky barriers, validating the hypothesis that the experimental set up deals with memristive behavior.

2) Two-Terminal Schottky-Barrier Silicon Nanowires After the Bio-Functionalization Process (Memristive Biosensors): The most innovative change introduced by the bio-functionalization is the wide difference in the bias voltage used for reaching current minima. This means that a voltage gap is created after the functionalization with biological molecules as a further memory effect on the voltage scan across the nanowire [16], [17].

A Schottky diode can also be described with an equivalent circuit model consisting of a non-linear capacitor in parallel to a non-linear resistor accordingly to literature [35]. The capacitor stands for the space charge capacitance and reflects only the free carriers of the material, while the resistor
Electrical characteristics: Bare Memristive Device

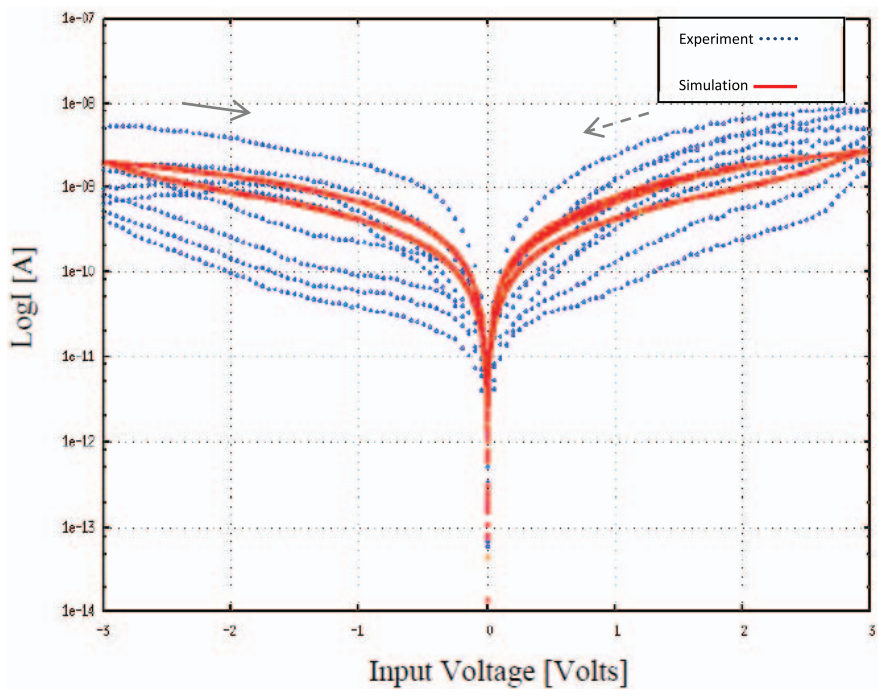

Fig. 5. Semi-logarithmic current to voltage curve presenting simulation results obtained from the equivalent circuit (Figure 4) comparing to experimental results coming from electrical measurements of bare memristive device. The simulation current is scaled accordingly to the experimental current range the input voltage amplitude is $[-3: 3]$ Volts and the frequency is set at $700 \mathrm{~Hz}$. The forward (solid line arrow) and backward (dashed line arrow) regimes are depicted in the figure. Since, by nature the devices usually present a non-identical behavior multiple experimental curves are presented and it is demonstrated that the computationally obtained results follow in good approximation the average behavior of the physical system, and it can be concluded that the experimental setup exhibits memristive behavior.

represents the residual conductance of the diode. In the case of lightly doped materials the free carrier concentration can become comparable to the deep level concentration and in this case charged and recharged deep levels also contribute considerably to the measured capacitance.

In a Schottky barrier, the barrier is high enough that there is a depletion region in the semiconductor near the interface. In the depletion region of the Schottky barrier, dopants remain ionized and give rise to the "space charge" which, in turn, gives rise to a capacitance of the junction. The metal-semiconductor interface and the opposite boundary of the depleted area act like two capacitor plates, with the depletion region acting as a dielectric. The amount of junction capacitance initially depends on the applied terminal voltages. By applying a voltage to the junction, the width of the space charge layer will be shifted and the space charge within the depletion region will vary, since additional defect centers will be ionized and as a result the capacitance will also be different. Furthermore, the charging and recharging of the trap levels during a measurement cycle periodically change the Schottky barrier height and finally, the modified measurement current gives a capacitive contribution to the diode admittance. Thus, both effects, variation of bias and consequently the ionization of traps, cause a change in the junction capacitance [36].

An equivalent circuit containing non-linear subcircuits (RC) consisting of a non-linear capacitor in parallel to a non-linear resistor is further then introduced (Figure 6) in order to correctly model the appearance of the voltage gap, after the bio-modification of the bare nanofabricated device with antibodies. The sub-circuits are connected in 


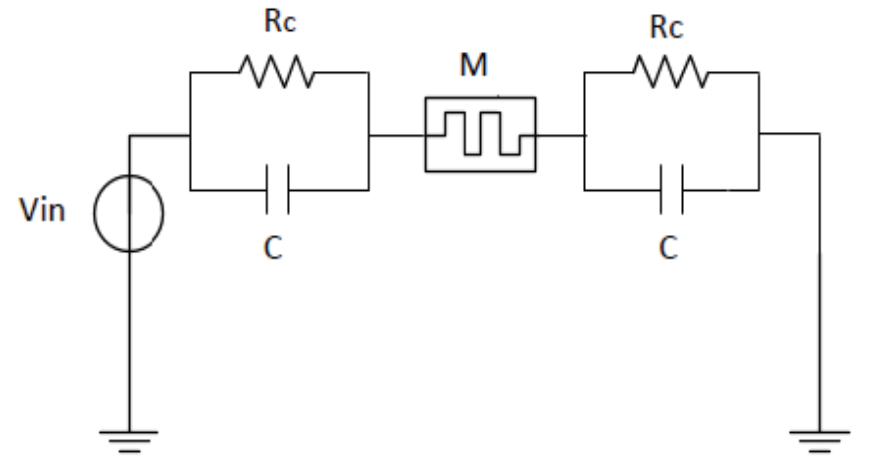

Fig. 6. Equivalent circuit of Memristive Biosensors. A sinusoidal input Voltage (Vin) source is connected in series with a memristor and with the non-linear sub-circuits (RC). The sub-circuits (RC) introduced consist of a non-linear capacitor in parallel to a non-linear resistor.

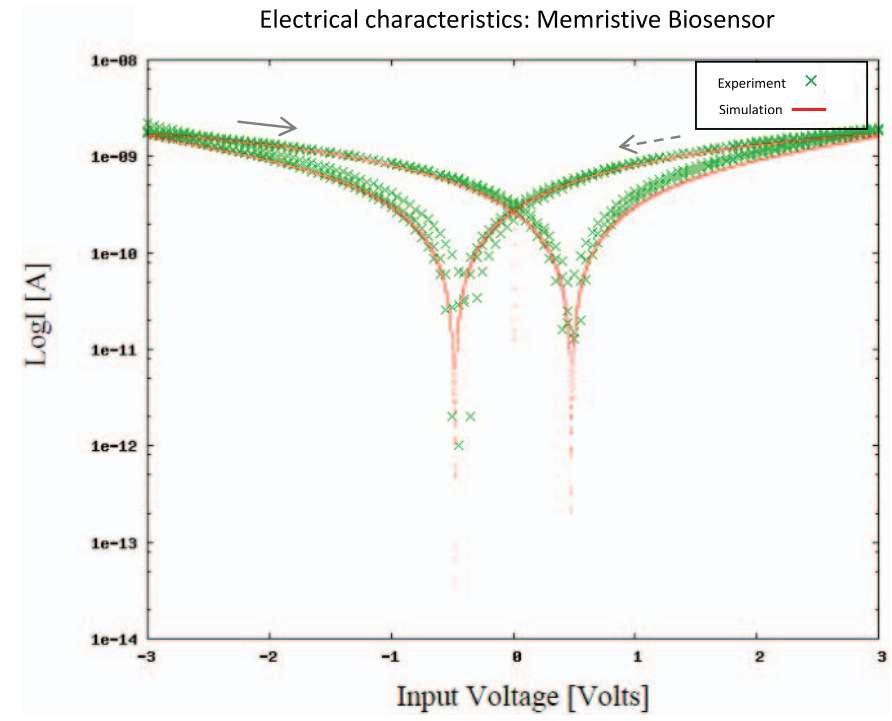

Fig. 7. Semi-logarithmic current to voltage curves depicting simulation results (red curve) obtained from the equivalent circuit, as compared to experimental results coming from electrical measurements for the case of memristive biosensor, namely, the nanofabricated device after the bio-functionalization with antibodies, for three voltage sweeps (green curves). The simulation current is scaled accordingly to the experimental current range. The input voltage amplitude is $[-3: 3]$ Volts and the frequency is set at $700 \mathrm{~Hz}$, and the resistances value at $0,85 \mathrm{k} \Omega$. The forward (solid line arrow) and backward (dashed line arrow) regimes are depicted in the figure. The two current minima for forward and backward regime are clearly separated occurring for different voltage values. The voltage gap can be clearly observed.

series to the memristor (M) of the initial equivalent circuit. It is demonstrated (Figure 7) that the two current minima are clearly separated and a voltage gap appears in the semi logarithmic current to voltage characteristics as due to the presence of the capacitors now introduced. The fitting of the simulation results with the experimental data confirms that the voltage gap appearing at the experimental current to voltage characteristics for the memristive biosensors can be also computationally reproduced successfully and fits in very good approximation the experimental outcomes.

Measurements of the junction capacitance is a very useful technique, giving information on Schottky barrier heights, dopant profiles, as well as the presence of traps and defects inside the semiconductor and at the interface [37].
Accumulating evidence from several works concerning relevant measurements [35]-[38] refers values for capacitances appearing in the junction area and refer to the space charge capacitance belonging in the range beginning of $\mathrm{pF}$ [35], [36] and reaching the values of $\mathrm{nF}$ [37], [38]. According to literature, the excess capacitance is a result of the combination in parallel of the space charge capacitance characterizing the diode and of the diffusion capacitances due to the minority carriers' injection. The reported values concerning the excess capacitance reach $43 \mathrm{nF}$ and it is considered that the excess capacitance mainly originates from the bulk Si rather than the interface of the diode in study [38] while typical capacitance values concerning only contributions by the depletion area are in the range of $\mathrm{pF}$ [35], [36]. It is worth mentioning that all the values found for the equivalent capacitance fit quite well the values reported in literature concerning the excess capacitance and thus, it is demonstrated that the presence of antibodies and thereafter of antigens on the memristive biosensor interacts deeply with the conductivity of the channel related to minority carriers.

\section{Impact of the Concentration of Antigens Uptaken After the Bio-Functionalization Process on the Voltage Gap}

The width of the simulated voltage gap can be modulated by the variation of the value of the capacitance introduced in the circuit. More specifically, it is observed that the two local minima are converging or shifted away the one from the other by modifying the input values of the capacitance introduced to the equivalent circuit.

Experimental observations [16], [17] identify a similar behavior of voltage gap modification with respect to the type and the concentration of the biological molecules uptaken on the device surface. More specifically, an enlargement of the hysteresis window due to the presence of positivelycharged molecules around the freestanding channel i.e. the antibodies after the bio-functionalization process is experimentally noticed. Accumulating data suggests that the maximum voltage gap observed is of a value of 1Volt approximately. In an antibody, it is considered that the positively charged residues are in excess comparing to the negatively charged ones. The surface charges on the sidewall of the nanowires influence the charge density in the channel. Consequently, the presence of antibodies all-around the nanowire contributes to extra positive charges surrounding the channel. The net contribution of their positively charges acts by creating an electrical field surrounding the channel of the device.

Furthermore it is found that positive electrical charges of antibodies, may act as a virtual all around gate [17], [39] giving similar effects that those appearing in in fully passivated nano-structures without any bio-functionalization but fabricated with an all-around inorganic gate. Thus, it can be concluded that the virtual positive all-around gate provided by positively charged proteins can play the role of a positive bias gate voltage affecting the device the same way as in the three terminal devices. The phenomenon can be related to the amount of charged Nickel atoms that are injected from the device terminals. The carriers' injection is modified by the applied bias electrical potential applied across the channel 
TABLE I

Voltage Gap Values Obtained Experimentally [17] For DifFEREnt Antigen ConCENTRATIOnS AND Computationally Estimated

Voltage Gap Values for Different Values of Capacitance, Selected According to the Experimental Data.

For 0fM Concentration of Antigens, it Is Considered That the Voltage Gap That Appears Is Created

ONLY DUE TO THE Bio-FunCTIONALIZATION With ANTIBODIES

\begin{tabular}{|c|c|c|c|}
\hline Antigen concentration [fM] & $\begin{array}{c}\text { Voltage gap [Volts] } \\
\text { Experimental values [17] }\end{array}$ & Capacitance (C) [nF] & $\begin{array}{c}\text { Voltage gap [Volts] } \\
\text { Simulation values }\end{array}$ \\
\hline \hline 0 & 0.84 & 36 & 0.844 \\
\hline 5 & 0.56 & 24 & 0.563 \\
\hline 10 & 0.37 & 15 & 0.362 \\
\hline
\end{tabular}

and as a result it affects the Schottky barrier at the interface accordingly to the well-known function of the nanowire FETs. However, the carriers' injection and consequently the Schottky barriers are further modified by the surface potential due to the antibodies added on the device surface. Consequently, it can be concluded that the surface potential of antibodies finally plays a significant role in the junction capacitance variation.

The presence of antigens on the device surface seems to demonstrate the opposite effects comparing to those resulting due to the presence of the antibodies. Antigens are considered to have a masking contribution to the presence of antibodies all-around the device and decrease the positive charge effect due to the presence of antibodies after the bio-functionalization process. Thus, the uptake of antigens acts by decreasing the value of the positive all around gate bias voltage created by the presence of antibodies. According to the previous arguments, the presence of antigens also affects the width of the voltage gap, which is already created by the presence of antibodies all around the device after bio-functionalization. Collectively, the experimental data depicts a contraction of the hysteresis window with increasing the concentration of antigens.

To further define the role of the capacitance value to the voltage gap, the experimentally obtained results concerning voltage gap values for different antigen concentrations [17] are taken into consideration and different capacitance values are introduced to the aforementioned equivalent circuit (Figure 6) designed for simulating the modified-memristive behavior, in order to reproduce computationally the voltage gap values obtained experimentally as reported in Table I. Furthermore the calibration curve (Figure 8) shows the computationally estimated voltage gap values that equal the values of the voltage gap obtained experimentally [17] for different antigen concentrations. The computationally obtained voltage gap values are a result of the different equivalent capacitance values introduced to the equivalent circuit and it is found that correspond to the values reported in literature for the excess capacitance [38]. Intermediate theoretical values for the voltage gap obtained from simulations for different capacitance values are also shown. It can be noticed that for achieving narrower voltage gaps lower values for the capacitance must be introduced to the equivalent circuit for reproducing computationally the corresponding experimental obtained voltage gap. This evidence suggest that increasing the concentration of antigens demands lower values for the capacitance introduced to the equivalent circuit in order to achieve the same value

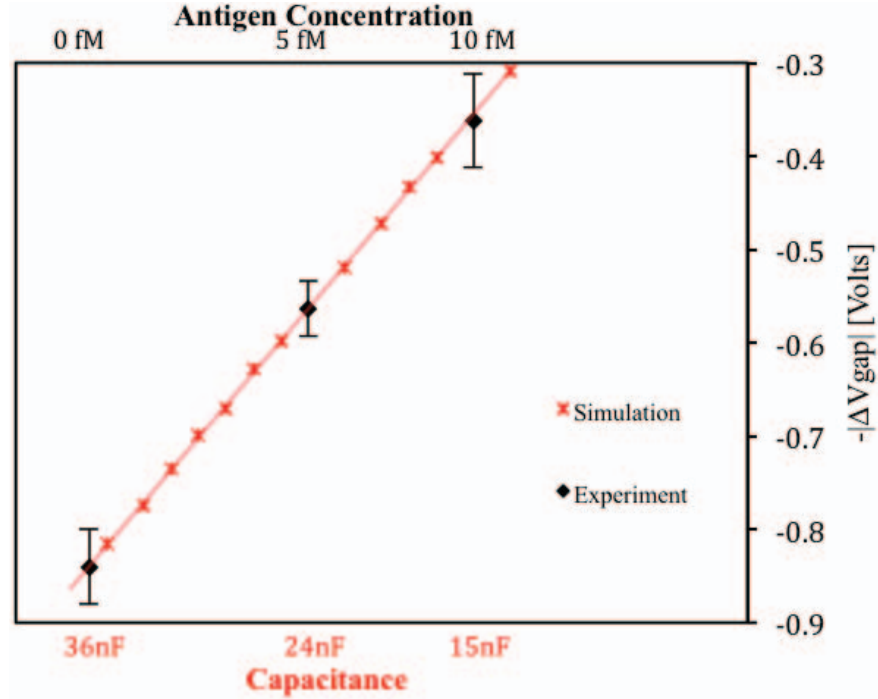

Fig. 8. Calibration curve obtained experimentally for three antigen concentrations [17]. The uptake with antigens modifies the memristive behavior such as $-|\Delta \mathrm{Vgap}|$ increases with the increase of the antigens' concentration. For OfM concentration of antigens, it is considered that the voltage gap that appears is created only due to the bio-functionalization with antibodies. The theoretical values for the voltage gap are results obtained from simulations for different capacitance values.

for the voltage gap, with respect to this range of capacitance values. For zero concentration of antigens (0fM) only the voltage gap already created by the presence of antibodies after the bio-functionalization process, is considered.

\section{CONCLUSIONS}

In this paper, a complete study is carried out targeting at the investigation of the electrical characteristics obtained from freestanding two-terminal memristive biosensors.

Throughout modeling and simulations, the computationally resulting electrical characteristics are studied aiming at the investigation of the relationship between the electrical behavior obtained experimentally from freestanding two-terminal Schottky-barrier silicon nanowire devices and the biosensing and in order to elicit a proper physical meaning. The experimental data indicates that the current to voltage characteristics corresponding to the bare nanofabricated devices present a pinched hysteretic loop at zero voltage. Still, when biological substances are present on the device surface, the hysteresis shows a voltage gap in the semi-logarithmic current to voltage curve. 
First, a macromodel of a memristor element is created and combined with analog circuit elements forming equivalent circuit models. The equivalent circuit models introduced in this study reproduce and emulate successfully the behavior of the physical system fitting in good approximation the experimental results for bare devices and for devices with bio-modified surface. Therefore, the equivalent circuit models presented in the current work offer a bridge between the experimental electrical characteristics from the nanofabricated wires and the theory of memristors.

For the case of bare two-terminal Schottky-barrier silicon nanowire devices, an equivalent circuit model is introduced consisting of the memristor sandwiched between two nonidentical head-to-head Schottky barriers. Each one of the two Schottky barriers is modeled as a sub-circuit consisting of a diode in parallel to a resistor. Throughout simulations and adequate fitting between the experimental and computational outcomes, the present study confirms that the electrical characteristics obtained from experimental measurements with freestanding two-terminal Schottky-barrier silicon nanowire devices exhibit hysteretic properties imputable to memristive devices. The electrical characteristics obtained computationally in the present work, validate the hypothesis that the experimental set up deals with memristive behavior and confirm the memristive devices.

Moreover, the acquisition of a voltage difference between the current minima of the forward and backward regimes in semi-logarithmic current to voltage curve acquired experimentally after the bio-functionalization of the devices surface is also computationally achieved. In addition, the voltage difference between the current minima can be accordingly modified expressing the different concentration of antigens uptaken on the device surface. Regarding the case of the devices with bio-modified surface, the equivalent circuit model consisting of a memristor sandwiched between the Schottky barriers, now expressed as non-linear sub-circuit models comprising a nonlinear capacitor in parallel to a non-linear resistor, is introduced. The present study demonstrates computationally that the behavior of the physical system with bio-modified surface is related to capacitive effects due to the minority carrier in the nanowire device. Moreover, it is demonstrated that those capacitive effects are strongly affected by the concentration of antigens uptaken on the device surface, evidence that implies the efficiency of the memristive biosensor. The computational study presented in the current work offers an extremely good link between the concepts of analog electronics and biosensing and confirms the use of memristive effect for biosensing applications, demonstrating the Memristive Biosensors. It is worth mentioning that the same nanofabricated devices are currently implemented for sensing different bio-molecules in different conditions. To this end further computational investigation will take place in the future for further investigation of the sensors physical system.

\section{ACKNOWLEDGEMENTS}

The authors gratefully acknowledge the CMi staff of EPFL, for assisting with technical advice throughout the fabrication process.

\section{REFERENCES}

[1] J. Wua and R. McCreery, "Solid-state electrochemistry in molecule/TiO molecular heterojunctions as the basis of the $\mathrm{TiO}_{2}$ "memristor," J. Electrochem. Soc., vol. 156, no. 1, pp. 29-37, 2009.

[2] L. Agapito, S. Alkis, J. Krause, and H.-P. Cheng, "Atomistic origins of molecular memristors," J. Phys. Chem. C, vol. 113, no. 48, pp. 20713-20718, 2009.

[3] D. Wang et al., "Transmembrane potential across single conical nanopores and resulting memristive and memcapacitive ion transport," J. Amer. Chem. Soc., vol. 134, no. 8, pp. 3651-3654, 2012.

[4] M. Krems, Y. Pershin, and M. Di Ventra, "Ionic memcapacitive effects in nanopores," Nano Lett., vol. 10, no. 7, pp. 2674-2678, 2010.

[5] M. Di Ventra, Y. Pershin, and L. Chua, "Circuit elements with memory: Memristors, memcapacitors, and meminductors," Proc. IEEE, vol. 97, no. 10, pp. 1717-1724, Oct. 2009.

[6] D. Strukov, G. Snider, D. Stewart, and S. Williams, "The missing memristor found," Nature, vol. 453, pp. 80-83, Mar. 2008.

[7] G. Rose, J. Rajendran, and H. Manem, R. Karri, and R. Pino, "Leveraging memristive systems in the construction of digital logic circuits," Proc. IEEE, vol. 100, no. 6, pp. 2033-2049, Jun. 2012.

[8] R. Waser and M. Aono, "Nanoionics-based resistive switching memories," Nature Mater., vol. 6, no. 11, pp. 833-840, 2007.

[9] Y. Pershin and M. Di Ventra, "Experimental demonstration of associative memory with memristive neural networks," Neural Netw., vol. 23, no. 7, pp. 881-886, Sep. 2010.

[10] G. Indiveri, B.-L. Barranco, R. Legenstein, G. Deligeorgis, and T. Prodromakis, "Integration of nanoscale memristor synapses in neuromorphic computing architectures," Nanotechnology, vol. 24, no. 38, 2013, Art. ID 384010.

[11] A. Gelencser, T. Prodromakis, C. Toumazou, and T. Roska, "Biomimetic model of the outer plexiform layer by incorporating memristive devices," Phys. Rev. E, vol. 85, no. 4, 2012, Art. ID 041918.

[12] D. Sacchetto, P. Gaillardon, M. Zervas, S. Carrara, G. De Micheli, and Y. Leblebici, "Applications of multi-terminal memristive devices: A review," IEEE Circuits Syst. Mag., vol. 13, no. 2, pp. 23-41, Second Quarter 2013.

[13] P.-E. Gaillardon, D. Sacchetto, S. Bobba, and Y. Leblebici, "GMS: Generic memristive structure for non-volatile FPGAs," in Proc. IFIP/IEEE Int. Conf. Very Large Scale Integr. (VLSI-SoC), Oct. 2012, pp. 94-98.

[14] N. Elfström, R. Juhasz, I. Sychugov, T. Engfeldt, A. Karlström, and J. Linnros, "Surface charge sensitivity of silicon nanowires: Size dependence," Nano Lett., vol. 7, no. 9, pp. 2608-2612, Aug. 2007.

[15] F. Ishikawa et al., "Label-free, electrical detection of the SARS virus n-protein with nanowire biosensors utilizing antibody mimics as capture probes," ACS Nano, vol. 3, no. 5, pp. 1219-1224, 2009.

[16] D. Sacchetto, M.-A. Doucey, G. De Micheli, Y. Leblebici, and S. Carrara, "New insight on bio-sensing by nano-fabricated memristors," BioNanoScience, vol. 1, nos. 1-2, pp. 1-3, Jun. 2011.

[17] S. Carrara, D. Sacchetto, M.-A. Doucey, C. Baj-Rossi, G. De Micheli, and Y. Leblebici, "Memristive-biosensors: A new detection method by using nanofabricated memristors," Sens. Actuators B, Chem., vols. 171-172, pp. 449-457, Aug./Sep. 2012.

[18] F. Puppo, M. Di Ventra, G. De Micheli, and S. Carrara, "Memristive sensors for $\mathrm{pH}$ measure in dry conditions," Surf. Sci., vol. 624, pp. 76-79, Jun. 2014.

[19] E. Stern, R. Wagner, F.-J. Sigworth, R. Breaker, T.-M. Fahmy, and M.-A. Reed, "Importance of the debye screening length on nanowire field effect transistor sensors," Nano Lett., vol. 7, no. 11, pp. 3405-3409, Oct. 2007.

[20] J. J. Yang, M. D. Pickett, X. Li, D. A. A. Ohlberg, D. R. Stewart, and S. Williams, "Memristive switching mechanism for metal/oxide/metal nanodevices," Nature Nanotechnol., vol. 3, no. 7, pp. 429-433, 2008.

[21] S. Shin, K. Kim, and S.-M. Kang, "Compact models for memristors based on charge-flux constitutive relationships," IEEE Trans. Computer-Aided Design Integr. Circuits Syst., vol. 29, no. 4, pp. 590-598, Apr. 2010.

[22] S. Benderli and T.-A. Wey, "On SPICE macromodelling of $\mathrm{TiO}_{2}$ memristors," Electron. Lett., vol. 45, no. 7, pp. 377-379, 2009.

[23] Z. Biolek, D. Biolek, and V. Biolkova, "SPICE model of memristor with nonlinear dopant drift," Radioengineering, vol. 18, no. 2, pp. 210-214, 2009.

[24] A. Rak and G. Cserey, "Macromodeling of the memristor in SPICE," IEEE Trans. Comput.-Aided Design Integr. Circuits Syst., vol. 29, no. 4, pp. 632-636, Apr. 2010. 
[25] Z. Biolek, D. Biolek, and V. Biolkova, "Pinched hysteretic loops of ideal memristors, memcapacitors and meminductors must be "self-crossing," Electron. Lett., vol. 47, no. 25, pp. 1385-1387, Dec. 2011.

[26] L. O. Chua, "Memristor-The missing circuit element," IEEE Trans. Circuit Theory, vol. 18, no. 5, pp. 507-519, Sep. 1971.

[27] L. Chua and S. Kang, "Memristive devices and systems," Proc. IEEE, vol. 64, no. 2, pp. 209-223, Feb. 1976.

[28] L. Chua, "Resistance switching memories are memristors," Appl. Phys. A, vol. 102, no. 4, pp. 765-783, 2011.

[29] S. Williams, "How we found the missing memristor," IEEE Spectr. vol. 45 , no. 12 , pp. $28-35$, Dec. 2008 .

[30] Y.-C. Tseng and J. Bokor, "Characterization of the metal-semiconductor carbon nanotube Schottky contact using capacitive methods," Appl. Phys. Lett., vol. 96, no. 1, p. 013103, 2010.

[31] S.-H. Lee, Y. Seop, S.-W. Hwang, and D. Ahn, "A SPICE-compatible new silicon nanowire field-effect transistors (SNWFETs) model," Trans. Nanotechnol., vol. 8, no. 5, pp. 643-649, Sep. 2009

[32] H. Elhadidy, J. Sikula, and J. Franc, "Symmetrical current-voltage characteristic of a metal-semiconductor-metal structure of Schottky contacts and parameter retrieval of a CdTe structure," Semicond. Sci. Technol., vol. 27, no. 1, p. 015006, 2012.

[33] S.-H. Lee, Y. Yu, S.-W. Hwang, and D. Ahn, "Equivalent circuit model of semiconductor nanowire diode by SPICE," J. Nanosci. Nanotechnol., vol. 7, no. 11, pp. 4089-4093, Nov. 2007.

[34] C.-Y. Yim et al., "Electrical properties of the $\mathrm{ZnO}$ nanowire transistor and its analysis with equivalent circuit model," J. Korean Phys. Soc., vol. 48, no. 6, pp. 1565-1569, 2006.

[35] K. Steiner, "Capacitance-voltage measurements on Schottky diodes with poor ohmic contacts," IEEE Trans. Instrum. Meas., vol. 42, no. 1, pp. 39-43, Feb. 1993.

[36] M. Bleicher and E. Lange, "Schottky-barrier capacitance measurements for deep level impurity determination," Solid State Electron., vol. 16, no. 3, pp. 375-380, Mar. 1973

[37] P. Ho, E. Yang, H. Evans, and X. Wu, "Electronic states at silicidesilicon interfaces," Phys. Rev. Lett., vol. 56, no. 2, pp. 177-180, 1986.

[38] J. Werner, A. F. J. Levi, R. T. Tung, M. Anzlowar, and M. Pinto, "Origin of the excess capacitance at intimate Schottky contacts," Phys. Rev. Lett., vol. 60 , no. 1 , pp. 53-56, 1988.

[39] Y. Cui, Q. H. Park, and C.-M. Lieber, "Nanowire nanosensors for highly sensitive and selective detection of biological and chemical species," Science, vol. 293, no. 5533, pp. 1289-1292, Aug. 2001.

Ioulia Tzouvadaki received the B.Sc. degree in physics from the National and Kapodistrian University of Athens, Greece, and the M.Sc. degree in microsystems and nanodevices from the National Technical University of Athens (NTUA). She is currently pursuing the Ph.D. degree in microsystems and microelectronics with the Integrated System Laboratory, EPFL, under the supervision of S. Carrara and G. De Micheli. Her master's thesis concerned the computational study and simulation, of polymer nanocomposite materials, with the Computational Materials Science and Engineering research Group, School of Chemical Engineering, NTUA. During her post-graduate studies, she was with the Clean Room Laboratory, National Center for Scientific Research Demokritos, and involved in experimental construction processes concerning integrated circuits and the experimental characterization process of nanomaterials and nanodevices. The main aim of the thesis is the labelfree detection of prostate cancer biomarkers, and includes the fabrication of memristive biosensors.

Francesca Puppo received the B.Sc. degree in biomedical engineering and the M.Sc. (cum laude) degree in bioengineering from the University of Genoa, Italy, in 2008 and 2011, respectively. She carried out the master's thesis with the Integrated System Laboratory (LSI), EPFL, Switzerland, in collaboration with the Department of Electrical Engineering and Biophysics, University of Genoa, Italy. She is currently pursuing the Ph.D. degree in microsystems and microelectronics with the Integrated System Laboratory (LSI), EPFL, under the supervision of S. Carrara and G. De Micheli. She worked on the design and the fabrication of a CNTFET-based biosensor. She also interned at LSI for a five-month project based on the study of silicon nanowire-based biosensors. Her research focuses on the design and fabrication of novel memristivebiosensor based on silicon nanowires.
Marie-Agnès Doucey received the (Hons.) degree in biochemistry from the Friedrich-Miescher Institute, Novartis, Basel, Switzerland, and the Engineering degree in biotechnology from the Ecole Supérieure de Biotechnologies de Strasbourg, France. She is currently a Project Leader with the Division of Experimental Oncology, University of Lausanne/CHUV, Lausanne. She was a Project Leader with the Division of Experimental Oncology, University of Lausanne/CHUV/CePO, and Maitreassistante, Institute of Biochemistry, Epalinges. She has over 20 publications in peer-reviewed international journals.

Giovanni De Micheli (F'94) is currently a Professor and the Director of the Institute of Electrical Engineering and the Integrated Systems Centre with EPFL, Switzerland. He is the Program Leader of the Nano-Tera.ch program. His research interests include several aspects of design technologies for integrated circuits and systems, such as synthesis for emerging technologies, networks on chips, and 3-D integration. He is also interested in heterogeneous platform design, including electrical components and biosensors, and data processing of biomedical information. He is a fellow of the Association for Computing Machinery and a member of Academia Europaea. He was a recipient of the 2003 IEEE Emanuel Piore Award for contributions to computer-aided synthesis of digital systems. He received the Golden Jubilee Medal for the outstanding contributions to the IEEE CAS Society in 2000. He received the 1987 D. Pederson Award for the best paper on the IEEE TRANSACTIONS ON COMPUTER-AIDED DESIGN at the International Centre for Applied Science, two best paper awards at the Design Automation Conference in 1983 and 1993, and a best paper award at the DATE Conference in 2005 .

Sandro Carrara received the $\mathrm{Ph} . \mathrm{D}$. degree in biochemistry and biophysics from the University of Padua, Italy, and the master's degree in Physics from the University of Genoa, Italy. He was a Professor of Optical and Electrical Biosensors with the Department of Electrical Engineering and Biophysics, University of Genoa, Italy, and a Professor of Nanobiotechnology with the University of Bologna, Italy. $\mathrm{He}$ is currently with EPFL, Lausanne, Switzerland, as a Faculty Member (MER). He has over 190 scientific publications and holds 12 patents. He has several Top-25 Hottest-Articles (2004, 2005, 2008, 2009, and two times in 2012) published in highly ranked international journals, such as Biosensors and Bioelectronics, Sensors and Actuators-Part B, the IEEE SENSORs Journal, and Thin Solid Films. From 1997 to 2000, he was a member of an International Committee at the ELETTRA Synchrotron in Trieste. He is a member of the Board of Governors of the IEEE Circuits and Systems Society. He is member at large of the IEEE Sensors Council. He is a founder and the Editor-in-Chief of the journal BioNanoScience by Springer, a Topical Editor of the IEEE SENSORS JOURNAL, and an Associate Editor of the IEEE TRANSACTIONS ON BIOMEDICAL CIRCUITS AND SYSTEMS. He has been appointed as a CASS Distinguished Lecturer for the years 2013-2014. His scientific interests are on electrical phenomena of nanobiostructured films, and include CMOS design of biochips based on proteins and DNA. His work received a NATO Advanced Research Award in 1996 for the original contribution to the physics of single-electron conductivity in nano-particles, two Best Paper Awards at the IEEE PRIME Conference in 2010 (Berlin), and in 2009 (Cork), a Best Poster Award at the Nanotera workshop in 2011 (Bern), and a Best Poster Award at the NanoEurope Symposium in 2009 (Rapperswil). $\mathrm{He}$ also received the Best Referees Award from the journal Biosensor and Bioelectronics in 2006. From 2000 to 2003, he was a Scientific Leader of a National Research Program in nanobiotechnology. He was an internationally esteemed expert of the evaluation panel of the Academy of Finland in a research program for the years 2010-2013. He has been the General Chairman of the IEEE BioCAS Conference in 2014. 